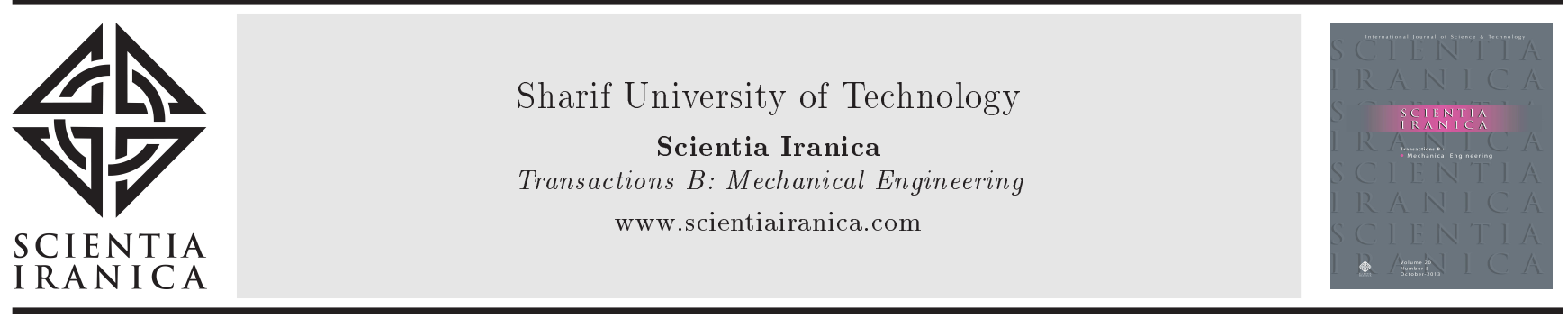

\title{
MHD boundary layer flow of an incompressible upper-convected Maxwell fluid by optimal homotopy asymptotic method
}

\author{
H. Ullah ${ }^{\mathrm{a}, *}$, S. Islam ${ }^{\mathrm{a}}$, I. Khan ${ }^{\mathrm{b}}$, S. Sharidan ${ }^{\mathrm{c}}$ and M. Fiza ${ }^{\mathrm{a}}$ \\ a. Department of Mathematics, Abdul Wali Khan University, Mardan, 23200, Pakistan. \\ b. College of Engineering, Majmaah University, Majmaah, Saudi Arabia. \\ c. Department of Mathematical Sciences, Faculty of Science, Universiti Teknologi Malayisa (UTM ), 81310 Skudai, Johar Bohru, \\ Johor Malaysia.
}

Received 14 November 2014; received in revised form 16 December 2015; accepted 10 May 2016

\section{KEYWORDS \\ OHAM; \\ MHD Maxwell fluid; \\ Boundary layer; \\ Non-linear boundary \\ value problem.}

\begin{abstract}
In this article, the magneto-hydrodynamics (MHD) boundary layer flow of an Upper-Convected Maxwell (UCM) fluid has been studied. The governing equations of the MHD boundary layer flow of UCM fluid have been reduced to nonlinear Ordinary Differential Equations (ODEs) by using similarity transformation. The basic idea of Optimal Homotopy Asymptotic Method (OHAM) for the nonlinear ODEs has been presented. The results obtained by OHAM have been compared with those of Homotpy Perturbation Method (HPM) and numerical Boundary Value Problem Method in order to verify accuracy of the proposed method. The effect of the Hartman and Deborah numbers has been discussed. It has been observed that with increase in Hartman number, velocity component steadily decreases and when increasing the magnetic force, thickness of the boundary layer decreases. The obtained solutions show that OHAM is an effective, simpler, easier, and explicit method.
\end{abstract}

(C) 2017 Sharif University of Technology. All rights reserved.

\section{Introduction}

Nonlinear phenomena play a significant role in applied mathematics, physics, and engineering sciences because most of the problems in these fields of study are nonlinear, particularly in the non-Newtonian MHD fluids. Few analytic methods have been presented as solutions to nonlinear problems. Most of the techniques like Adomian Decomposition Method (ADM) [1], Variational Iteration Method (VIM) [2], Differential Transform Method (DTM) [3], Homotopy Perturbation Method (HPM) [4], and Radial Basis Function (RBF) [5] are used as solutions to weakly nonlinear problems and they are limited for strongly nonlinear problems.

\footnotetext{
*. Corresponding author.

E-mail address: hakeemullah1@gmail.com (H. Ullah)
}

Perturbation Methods [6-8] have been studied as the solution to strongly nonlinear problems. These methods embrace a small parameter, which cannot be found easily. New analytic methods, such as Artificial Parameters Method (APM) [9], Homotopy Analysis Method (HAM) [10], and Homotopy Perturbation Method (HPM) [4], have been introduced for small parameters. These methods pool the homotopy with the perturbation techniques. Recently, Marinca et al. introduced Optimal Homotopy Asymptotic Method (OHAM) [11-15] for the solution of nonlinear problems, which made perturbation methods independent of the assumption of small parameters.

MHD fluid studies the motion of electrically conducting fluid in the presence of magnetic field. This area of study was first introduced by Alfven [16] and is important in plasma physics, astrophysics, and engi- 
neering. Maxwell model is one of the non-Newtonian fluid models having the relaxation phenomena. This type of fluid has much application in viscoelastic problems which contain small dimensionless relaxation time [17-19]. Most of the MHD problems are nonlinear and strongly nonlinear. An enormous amount of research work has been invested in the study of nonlinear problems [20-24]. In this article, we shall deal with the MHD Maxwell flow problem, a strongly nonlinear boundary value problem [25]. Recently, some of the authors have studied effect of MHD on UCM fluid with heat and mass transfer [26,27].

The motivation of this paper is to apply OHAM for the solution of MHD Maxwell flow problem. In [1115], OHAM has been proved to be useful for obtaining an approximate solution of nonlinear boundary value problems. In this work, we have proved that OHAM is also useful and reliable for the solution of MHD Maxwell flow problems equation, hence, showing its validity and great potential as the solution to transient physical phenomenon in science and engineering.

In the succeeding section, the basic idea of OHAM [11-15] is formulated for the solution of boundary value problems. In Section 3, the effectiveness of the formulation of OHAM for MHD Maxwell flow problem has been studied.

\section{Fundamental mathematical theory of OHAM}

Let us see the OHAM in the following general nonlinear differential equation:

$$
\mathcal{L}(f(x))+h(x)+\mathcal{N}(f(x))=0,
$$

along with boundary conditions of the form:

$$
\mathcal{B}\left(f, \frac{d f}{d x}\right)=0
$$

where $\mathcal{L}$ is the linear operator, $f(x)$ is an unknown function, $h(x)$ is a known function, $\mathcal{N}(f(x))$ is a nonlinear differential operator, and $\mathcal{B}$ is a boundary operator.

According to OHAM, one can construct an optimal homotopy $\phi(x, r): \Omega \times[0,1] \rightarrow \Re$, which satisfies:

$$
\begin{aligned}
& (1-r)[\mathcal{L}(\phi(x, r))+h(x)] \\
& =H(r)[\mathcal{L}(\phi(x, r))+h(x)+\mathcal{N}(\phi(x, r))] \\
& \mathcal{B}\left(\phi(x, r), \frac{\partial \phi(x, r)}{\partial x}\right)=0
\end{aligned}
$$

where $r \in[0,1]$ is an embedding parameter, $\phi(x, r)$ is an unknown function, and $H(r)$ is a nonzero auxiliary function. The auxiliary function $H(r)$ is nonzero for $r \neq 0$ and $H(0)=0$. Eq. (3) is the mathematical structure of OHAM homotopy.

It is defined that:

$$
r=0 \Rightarrow \phi(x, 0)=f_{0}(x)
$$

and:

$$
r=1 \Rightarrow \phi(x, 1)=f(x),
$$

respectively. Thus, as $r$ varies from 0 to 1 , the solution $\phi(x, r)$ varies from $f_{0}(x)$ to $f(x)$, where $f_{0}(x)$ is obtained from Eqs. (1) and (2) for $r=0$ :

$$
\mathcal{L}\left(f_{0}(x)\right)+h(x)=0, \quad \mathcal{B}\left(f_{0}, \frac{d f_{0}}{d x}\right)=0 .
$$

Next, we choose auxiliary function $H(r)$ in the form:

$$
H(r)=r C_{1}+r^{2} C_{2}+r^{3} C_{3}+\cdots,
$$

where $C_{1}, C_{2}, C_{3}, \cdots$ are constants and can be found later.

To obtain an approximate solution, we expand $\phi\left(x, r, C_{i}\right)$ by Taylor's series about $r$ in the following form:

$$
\begin{aligned}
& \phi\left(x, r, C_{1}, C_{2}, \cdots, C_{i}\right)=f_{0}(x) \\
& +\sum_{k=1}^{\infty} f_{k}\left(x, C_{1}, C_{2}, \cdots, C_{i}\right) r^{k}, \quad i=1,2, \cdots
\end{aligned}
$$

Now, substituting Eq. (9) into Eqs. (1) and (2) and equating the coefficient of like powers of $r$, we obtain the zeroth-order problem given by Eq. (7); the firstand second-order problems are given by Eqs. (10) and (12), respectively, and the general governing equations for $f_{k}(x)$ are given by Eq. (12):

$$
\begin{aligned}
\mathcal{L}\left(f_{1}(x)\right)= & C_{1} \mathcal{N}_{0}\left(f_{0}(x)\right), \\
\mathcal{B}\left(f_{1}, \frac{d f_{1}}{d x}\right)=0 & \\
\mathcal{L}\left(f_{2}(x)\right)- & \mathcal{L}\left(f_{1}(x)\right) \\
= & C_{1} \mathcal{N}_{0}\left(f_{0}(x)\right)+C_{1}\left[\mathcal{L}\left(f_{1}(x)\right)\right. \\
& \left.+\mathcal{N}_{1}\left(f_{0}(x), f_{1}(x)\right)\right] \\
\mathcal{B}\left(f_{2}, \frac{d f_{2}}{d x}\right) & =0, \\
\mathcal{L}\left(f_{k}(x)\right)- & \mathcal{L}\left(f_{k-1}(x)\right) \\
= & C_{k} \mathcal{N}_{0}\left(f_{0}(x)\right)+\sum_{i=1}^{k-1} C_{i}\left[\mathcal{L}\left(f_{k-i}(x)\right)\right. \\
& \left.+\mathcal{N}_{k-i}\left(f_{0}(x), f_{1}(x), \cdots, f_{k-i}(x)\right)\right], \\
\mathcal{B}\left(f_{k}, \frac{d f_{k}}{d x}\right) & =0, \quad k=2,3, \cdots,
\end{aligned}
$$


where $\mathcal{N}_{k-i}\left(f_{0}(x), f_{1}(x), \cdots, f_{k-i}(x)\right)$ is the coefficient of $r^{k-i}$ in the expansion series of $\mathcal{N}(\phi(x, r))$ about the embedding parameter $r$.

$$
\begin{aligned}
\mathcal{N}\left(\phi\left(x, r, C_{i}\right)\right) & =\mathcal{N}_{0}\left(f_{0}(x)\right) \\
& +\sum_{k \geq 1} N_{k}\left(f_{0}, f_{1}, f_{2}, \ldots, f_{k}\right) r^{k}, \quad i=1,2,3, \ldots
\end{aligned}
$$

It should be underscored that $f_{k}$ for $k \geq 0$ is governed by the linear equations with linear boundary conditions that come from the original problem, which can be easily solved.

It has been observed that the convergence of the series in Eq. (9) depends on the auxiliary constants $C_{1}, C_{2}, \cdots$. If it is convergent at $r=1$, one has:

$\tilde{u}\left(x, C_{1}, C_{2}, \cdots C_{i}\right)=f_{0}(x)+\sum_{k \geq 1} f_{k}\left(x, C_{1}, C_{2}, \cdots C_{i}\right)$.

Substituting Eq. (14) into Eq. (1), the following expression for residual can be obtained:

$$
\begin{aligned}
& R\left(x, C_{1}, C_{2}, \cdots C_{i}\right)=\mathcal{L}\left(\tilde{f}\left(x, C_{1}, C_{2}, \cdots C_{i}\right)\right) \\
& +h(x)+\mathcal{N}\left(\tilde{f}\left(x, C_{1}, C_{2}, \cdots C_{i}\right)\right) .
\end{aligned}
$$

If $R\left(x, C_{1}, C_{2}, \cdots, C_{i}\right)=0$, then $\tilde{f}\left(x, C_{1}, C_{2}, \cdots, C_{i}\right)$ is the exact solution of the problem. Generally, it does not happen, especially in nonlinear problems.

For determination of auxiliary constants, $C_{i}, i=$ $1,2, \cdots, m$, there are different methods like Galerkin's method, Ritz method, Least Squares method, and Collocation method. One can apply the method of Least Squares as follows:

$$
J\left(C_{1}, C_{2}, \cdots, C_{m}\right)=\int_{a}^{b} R^{2}\left(x, C_{1}, C_{2}, C_{3} \cdots, C_{m}\right) d x
$$

where $a$ and $b$ are two values depending on the nature of the given problem.

The auxiliary constants $C_{i}, i=1,2, \cdots, m$, can be optimally found from Eq. (17):

$$
\frac{\partial J}{\partial C_{1}}=\frac{\partial J}{\partial C_{2}}=\cdots=\frac{\partial J}{\partial C_{m}}=0
$$

The $m$ th-order approximate solution can be obtained by these optimal constants. The constant $C_{i}$ can also be determined by another method as follows:

$$
\begin{gathered}
R\left(k_{1}, C_{1}, C_{2}, \cdots, C_{m}\right)=R\left(k_{2}, C_{1}, C_{2}, \cdots, C_{m}\right) \\
=\cdots=R\left(k_{m}, C_{1}, C_{2}, \cdots, C_{m}\right)=0 \\
i=1,2, \cdots, m
\end{gathered}
$$

The convergence of OHAM is directly proportional to the number of optimal constants $C_{1}, C_{2}, \cdots$, which is determined by Eqs. (16) and (17).

It is easy to observe [13] that the Homotopy Perturbation Method (HPM) proposed by He [4] is a special case of Eq. (3) when $H(r)=-r$. Moreover, the Homotopy Analysis Method (HAM) proposed by Liao [11] is another special case of Eq. (3) when $H(r)=$ $r \hbar$, where $\hbar$ is chosen from " $\hbar$-curves" [12].

\section{Problem formulation and solution}

To demonstrate effectiveness of the OHAM formulation, consider the two-dimensional flow of an incompressible upper-convected Maxwell fluid. A uniform static magnetic field, $B_{0}$, is applied perpendicular to the direction of flow. The continuity equation of the flow is [25]:

$$
\frac{\partial u}{\partial \bar{x}}+\frac{\partial v}{\partial \bar{y}}=0
$$

The Cauchy Stress tensor, $T$, for the Maxwell fluid is expressed as [26]:

$$
T=-p I+S,
$$

where the extra stress tensor, $S$, is calculated [26] by:

$$
S+\lambda\left(\frac{d S}{d t}-L S-S L^{T}\right)=\mu A_{1} .
$$

In Eq. (21), $\mu$ is the viscosity, $\lambda$ is relaxation time, and the first Rivlin-Ericksen tensor, $A_{1}$, is given by [28]:

$$
A_{1}=\nabla V+(\nabla V)^{T}
$$

The momentum differential equation for this flow is given by:

$$
\rho \frac{d V}{d t}=f_{b}-\nabla p+\mu \nabla^{2} V
$$

where $\rho$ is density, $f_{b}$ is body force, and $p$ is the pressure. The magnetic force in the $x$ direction is given by Lorenz force [29]:

$$
F_{\mathrm{L}}=J \times B
$$

where $F_{\mathrm{L}}$ is the Lorentz force, $J$ is electric current density, and $B$ is magnetic flux. The current density is obtained by Ohm's law [29]:

$$
J=\sigma(E+V \times B),
$$

where $\sigma$ is electric conductivity and $E$ is electric field. A magnetic field in the medium is induced by the motion of electrically conducting fluid in an applied magnetic field, known as induced magnetic field. The 
total magnetic field comprises the applied and induced magnetic fields. The induced magnetic field may be neglected at small magnetic Reynolds numbers [30]. According to the mentioned assumption, the electric field is zero because the external polarization of ionized fluid is assumed to be zero. $E=0$ in Eqs. (24) and (25) reduces them to:

$$
F_{\mathrm{L}}=-\sigma B_{o}^{2} V \text {. }
$$

The steady momentum equation is given by:

$$
\begin{aligned}
& u \frac{\partial u}{\partial \bar{x}}+v \frac{\partial u}{\partial \bar{y}}=-\frac{1}{\rho} \frac{\partial p}{\partial \bar{x}}-\frac{\sigma B_{0}^{2}}{\rho} u+\frac{1}{\rho}\left(\frac{\partial S_{\overline{x x}}}{\partial \bar{x}}+\frac{\partial S_{\overline{y y}}}{\partial \bar{y}}\right), \\
& u \frac{\partial v}{\partial \bar{x}}+v \frac{\partial v}{\partial \bar{y}}=-\frac{1}{\rho} \frac{\partial p}{\partial \bar{y}}+\frac{1}{\rho}\left(\frac{\partial S_{\overline{y x}}}{\partial \bar{x}}+\frac{\partial S_{\overline{y y}}}{\partial \bar{y}}\right)
\end{aligned}
$$

where $S_{\overline{x x}}, S_{\overline{x y}}, S_{\overline{y x}}$, and $S_{\bar{y} \bar{y}}$ are the components of extra stress tensor. In the absence of pressure gradient and boundary layer approximation [25], we have:

$$
\begin{aligned}
u \frac{\partial u}{\partial \bar{x}} & +v \frac{\partial u}{\partial \bar{y}}+\lambda\left[u^{2} \frac{\partial^{2} u}{\partial \bar{x}^{2}}+v^{2} \frac{\partial^{2} v}{\partial \bar{y}^{2}}+2 u v \frac{\partial^{2} u}{\partial \bar{x} \partial \bar{y}}\right] \\
& =v \frac{\partial^{2} u}{\partial \bar{y}^{2}}-\frac{\sigma B_{0}^{2}}{\rho} u
\end{aligned}
$$

where $v$ is kinematic viscosity. The boundary conditions for the problem are [25,28]:

$$
u(0)=U, \quad v(0)=0, \quad \text { and } \quad u(\infty)=0 .
$$

Using the stream functions $u=\frac{\partial \psi}{\partial \bar{y}}$ and $v=-\frac{\partial \psi}{\partial \bar{x}}$, Eq. (19) is identically satisfied and Eq. (29) is reduced to:

$$
\begin{gathered}
\frac{\partial \psi}{\partial \bar{y}} \frac{\partial^{2} \psi}{\partial \bar{x} \partial \bar{y}}-\frac{\partial \psi}{\partial \bar{x}} \frac{\partial^{2} \psi}{\partial \bar{y}^{2}}=v \frac{\partial^{3} \psi}{\partial \bar{y}^{3}}-\frac{\sigma B_{0}^{2}}{\rho} \frac{\partial \psi}{\partial \bar{y}} \\
-\lambda\left[\left(\frac{\partial \psi}{\partial \bar{y}}\right)^{2} \frac{\partial^{3} \psi}{\partial \bar{x}^{2} \partial \bar{y}}+\left(\frac{\partial \psi}{\partial \bar{x}}\right)^{2} \frac{\partial^{3} \psi}{\partial \bar{y}^{3}}\right. \\
\left.-2 \frac{\partial \psi}{\partial \bar{y}} \frac{\partial \psi}{\partial \bar{x}} \frac{\partial^{3} \psi}{\partial \bar{x} \partial \bar{y}^{2}}\right] .
\end{gathered}
$$

Upon using the transformations $x=\sqrt{\frac{U}{v \bar{x}}} \bar{y}$ and $\psi=$ $\sqrt{v \bar{x} U} f(x)$, Eqs. (31) becomes:

$$
2 f^{\prime \prime \prime}-2 M^{2} f^{\prime}+f f^{\prime \prime}-\beta\left(2 f f^{\prime} f^{\prime \prime}+f^{2} f^{\prime \prime \prime}+x f^{\prime 2} f^{\prime \prime}\right)=0,
$$

and boundary conditions become:

$$
f(0)=0, \quad f^{\prime}(0)=1, \quad \text { and } \quad f^{\prime}(\infty)=0 .
$$

In Eq. (32), $M^{2}=\frac{\sigma B_{0}^{2}}{\rho U}$ and $\beta=\frac{\lambda U}{2 x}$ are the Hartman number and Deborah number, and $f^{\prime}, f^{\prime \prime}$, and $f^{\prime \prime \prime}$ denotes the first, second, and third derivatives of $f$ with respect to $x$.
Applying the method formulation mentioned in Section 2 leads to the following:

\section{Zeroth-order problem:}

$$
\begin{aligned}
& f_{0}^{\prime \prime \prime}-2 M^{2} f_{0}^{\prime}=0, \\
& f_{0}(0)=0, \quad f_{0}^{\prime}(0)=1, \quad \text { and } \quad f_{0}^{\prime}(\infty)=0,
\end{aligned}
$$

from which we obtain:

$$
f_{0}(x)=\frac{\cosh (4 M)-\cosh (M(-4+x)) \cosh (4 M)}{M} .
$$

\section{First-order problem:}

$$
\begin{aligned}
& 2 f_{1}^{\prime \prime \prime}-2 M^{2} f_{0}^{\prime}+2 M^{2} C_{1} f_{0}^{\prime}-2 M^{2} f_{1}^{\prime}-C_{1} f_{0} f_{0}^{\prime \prime} \\
& \quad+2 \beta C_{1} f_{0} f_{0}^{\prime} f_{0}^{\prime \prime}+\zeta \beta C_{1} f_{0}^{\prime 2} f_{0}^{\prime \prime}-2 f_{0}^{\prime \prime \prime}-2 C_{1} f_{0}^{\prime \prime \prime} \\
& \quad+\beta C_{1} f_{0}^{2} f_{0}^{\prime \prime \prime}=0 \\
& \quad \\
& f_{1}(0)=0, \quad f_{1}^{\prime}(0)=0, \quad \text { and } \quad f_{1}^{\prime}(\infty)=0
\end{aligned}
$$

Its solution is:

$$
\begin{aligned}
& f_{1}(x)=\frac{1}{\left(576\left(-1+e^{8 M}\right)^{4} M^{3}\right)} e^{-3 M x} \\
& \left(576 e^{2 M x}\left(-1+e^{8 M}\right)^{3}\left(e^{8 M x}-e^{M x}\right)\left(-1+e^{M x}\right) M^{2}\right. \\
& +\left(-384 e^{4 M(3+x)}+768 e^{3 M(4+x)}+384 e^{4 M(5+x)}\right. \\
& -384 e^{2 M(6+x)}+384 e^{2 M(10+x)}+96 e^{M(24+x)} \\
& -768 e^{M(20+3 x)}-96 e^{M(8+5 x)}+e^{32 M}(23-12 M x) \beta \\
& +e^{24 M}(23-12 M x) \beta+e^{6 M x}(23+12 M x) \beta \\
& +e^{(8 M+6 M x)}(23+12 M x) \beta+48 e^{M(16+x)}(-1+4 \beta) \\
& +48 e^{(8 M+6 M x)}(23+12 M x) \beta \\
& +48 e^{M(16+5 x)}(-1+4 \beta) \\
& +48 e^{(8 M+6 M x)}(23+12 M x) \beta \\
& +48 e^{5 M x}(-1+4 \beta)+48 e^{M(16+x)}(-1+4) \beta \\
& +48 e^{M(32+x)}(1+4 \beta)-2 e^{3 M x}(48+5 \beta) \\
& +2 e^{M(32+3 x)}(48+5 \beta) \\
& +16 e^{3 M(8+x)}(12-36 M(x-4 \beta)+17 \beta) \\
& +576 e^{M(16+3 x)} M(2 x+23 \beta)
\end{aligned}
$$




$$
\begin{aligned}
& +24 e^{4 M(2+x)}(22+6 M x+17 \beta \\
& +3 M(-16+x(-11+M x) \beta) \\
& +24 e^{2 M(12+x)}(22-6 M x+17 \beta \\
& +3 M(16+x(11+M x)) \beta) \\
& -3 e^{2 M(16+x)}(16-53 \beta+48 M x(1+\beta) \\
& -3 e^{4 M x}(-16+53 \beta+48 M x(1+\beta) \\
& -24 e^{4 M(4+x)}(-6 M x+3 M(92 \\
& +x(-11+M x) \beta+17(2+\beta)) \\
& -24 e^{2 M(8+x)}(6 M x+3 M(-92 \\
& +x(11+M x) \beta+17(2+\beta) \\
& +3 e^{2 M(4+2 x)}(-80-53 \beta+48 M(-x \\
& +(-8+x) \beta))+3 e^{4 M(6+x)}(80+53 \beta \\
& +48 M(-x+(-8+x) \beta) C_{1} . \\
& +8 x^{2}+\beta
\end{aligned}
$$

Adding Eqs. (36) and (39), we obtain:

$$
\begin{aligned}
& f(x)=\frac{\cosh (4 M)-\cosh (M(-4+x)) \cosh (4 M)}{M} \\
& +\frac{1}{\left(576\left(-1+e^{8 M}\right)^{4} M^{3}\right)} e^{-3 M x}\left(576 e^{2 M x}\right. \\
& \left(-1+e^{8 M}\right)^{3}\left(e^{8 M x}-e^{M x}\right)\left(-1+e^{M x}\right) M^{2} \\
& +\left(-384 e^{4 M(3+x)}+768 e^{3 M(4+x)}+384 e^{4 M(5+x)}\right. \\
& -384 e^{2 M(6+x)}+384 e^{2 M(10+x)}+96 e^{M(24+x)} \\
& -768 e^{M(20+3 x)}-96 e^{M(8+5 x)}+e^{32 M}(23-12 M x) \beta \\
& +e^{24 M}(23-12 M x) \beta+e^{6 M x}(23+12 M x) \beta \\
& +e^{(8 M+6 M x)}(23+12 M x) \beta+48 e^{M(16+x)}(-1+4 \beta) \\
& +48 e^{(8 M+6 M x)}(23+12 M x) \beta \\
& +48 e^{M(16+5 x)}(-1+4 \beta) \\
& +48 e^{(8 M+6 M x)}(23+12 M x) \beta \\
& +48 e^{5 M x}(-1+4 \beta)+48 e^{M(16+x)}(-1+4) \beta \\
& +48 e^{M(32+x)}(1+4 \beta)-2 e^{3 M x}(48+5 \beta)
\end{aligned}
$$

$$
\begin{aligned}
& +2 e^{M(32+3 x)}(48+5 \beta)+16 e^{3 M(8+x)}(12 \\
& -36 M(x-4 \beta)+17 \beta) \\
& +576 e^{M(16+3 x)} M(2 x+23 \beta)+24 e^{4 M(2+x)}(22 \\
& +6 M x+17 \beta+3 M(-16+x(-11+M x) \beta) \\
& +24 e^{2 M(12+x)}(22-6 M x+17 \beta+3 M(16 \\
& +x(11+M x)) \beta)-3 e^{2 M(16+x)}(16-53 \beta \\
& +48 M x(1+\beta)-3 e^{4 M x}(-16+53 \beta \\
& +48 M x(1+\beta)-24 e^{4 M(4+x)}(-6 M x+3 M(92 \\
& +x(-11+M x) \beta+17(2+\beta)) \\
& -24 e^{2 M(8+x)}(6 M x+3 M(-92 \\
& +x(11+M x) \beta+17(2+\beta)-3 e^{2 M(4+2 x)}(-80 \\
& -53 \beta+48 M(-x+(-8+x) \beta))+3 e^{4 M(6+x)}(80 \\
& +53 \beta+48 M(-x+(-8+x) \beta) C_{1} .
\end{aligned}
$$

For computation of constant $C_{1}$, by applying the method of collocation, we get:

$$
\begin{aligned}
& C_{1}=-1.0535165176571712, \\
& \text { for } \quad \beta=0.4 \quad \text { and } \quad M=1 .
\end{aligned}
$$

Putting these values in Eq. (40), we obtain approximate solution of the form by Eq. (41) as shown in Box I. Solution of the problem by HPM [25] is obtained by Eq. (42) as shown in Box II.

\section{Results and discussions}

The formulation presented in Section 2 provides highly accurate solutions for the problems demonstrated in Section 3. We have used Mathematica 7 for most of our computational work. In Table 1, we have presented OHAM solution for different values of $x$. This table shows that the results obtained by OHAM are in excellent agreement with the results obtained by HPM and Numerical BVP method. It is found that the method presented in this work is very efficient and provides consistent values that optimize numerical methods with which it is compared. Figure 1 shows the variation of $f(x)$ against $x$ for different values of $M$ at fixed value of Hartman number, $\beta=0.5$. Also, Figure 2 shows the variation of $f^{\prime}(x)$ with respect to $x$ for different values of $M$ at $\beta=0.5$. For small values of the Hartman number, the boundary layer thickness 


$$
f(x)=e^{-3 x}\left[\begin{array}{l}
-0.0168439+0.00878815 x+e^{x}\left(0.228509+5.94248 \times 10^{-9} e^{4 x}\right. \\
+e^{5 x}\left(-6.35882 \times 10^{-13}-3.31764 \times 10^{-13} x\right)+e^{2 x}(0.81678+0.000353653 x) \\
+5.93385 \times 10^{-9} e^{3 x}(-4.57533+x)(8934.45+x) \\
\left.+0.0000176886 e^{x}(-2.78358+x)(20882.5+x)\right)
\end{array}\right]
$$

Box I

$$
\begin{aligned}
f(x)= & \frac{e^{-4 M}+e^{4 M}-e^{M(-4+x)}-e^{-M(-4+x)}}{M\left(e^{4 M}-e^{-4 M}\right)} \\
& -\frac{1}{4\left(-e^{28 M}+4 e^{20 M}-6 e^{12 M}+4 e^{4 M}-e^{-4 M}\right)\left(e^{8 M}-1\right) M} \int_{0}^{x} \cdots d x .
\end{aligned}
$$

Box II

Table 1. Comparison of OHAM with HPM and numerical results.

\begin{tabular}{cccc}
\hline & & $\begin{array}{c}\text { Numerical } \\
\text { results method } \\
{[\mathbf{2 5}]}\end{array}$ & OHAM \\
\hline 0 & 0.00000 & 0.000000 & 0.000000 \\
0.5 & 0.38662 & 0.386325 & 0.386258 \\
1.0 & 0.60613 & 0.605299 & 0.604764 \\
1.5 & 0.72485 & 0.724257 & 0.722115 \\
2.0 & 0.78562 & 0.786745 & 0.781490 \\
2.5 & 0.81461 & 0.818662 & 0.809267 \\
3.0 & 0.82698 & 0.834332 & 0.820713 \\
3.5 & 0.83119 & 0.841253 & 0.824337 \\
4.0 & 0.83192 & 0.843124 & 0.824854 \\
\hline
\end{tabular}

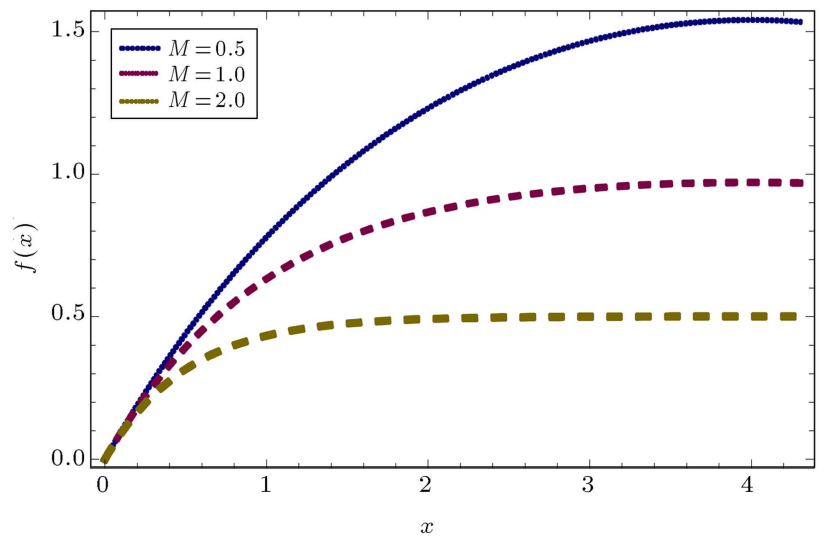

Figure 1. Effects of the Hartman number on $f(x)$ when $\beta=0.5$.

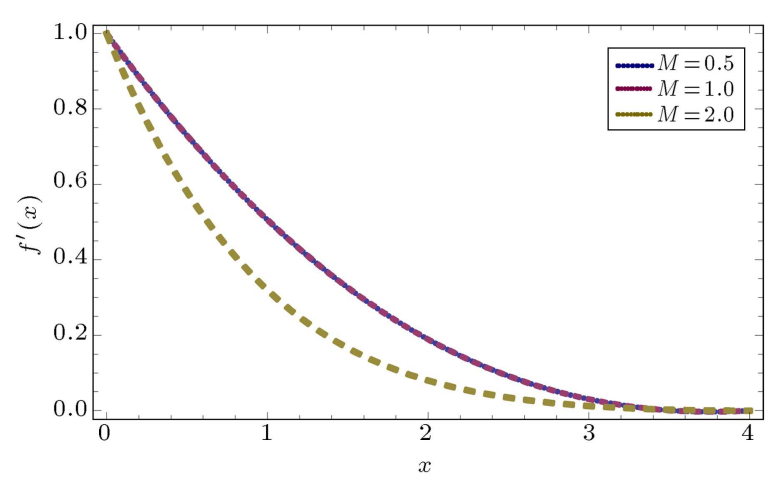

Figure 2. Effects of the Hartman number on $f^{\prime}(x)$ when $\beta=0.5$.

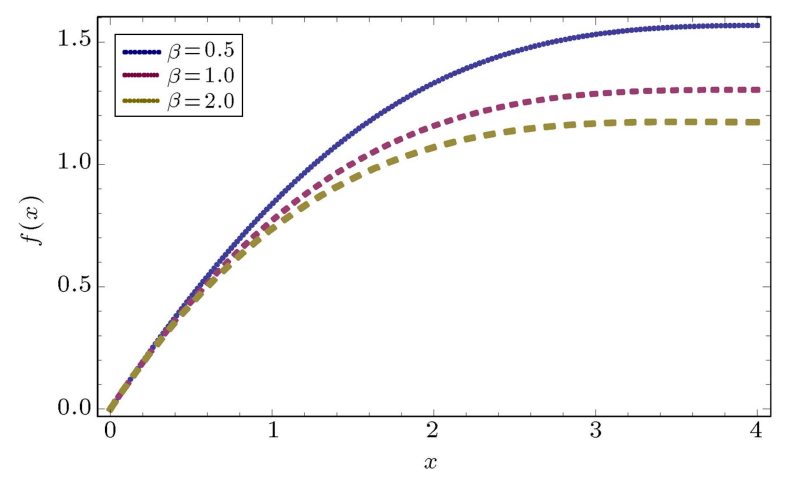

Figure 3. Effects of the Deborah number on $f(x)$ when $M=0.5$.

is larger and for large values of Hartman number, the boundary layer thickness is smaller. Figure 3 shows the variation of $f(x)$ with respect to $x$ for different values of $\beta$ at constant value of $M=0.5$, while Figure 4 represents the variation of $f^{\prime}(x)$ with respect to $x$ for 


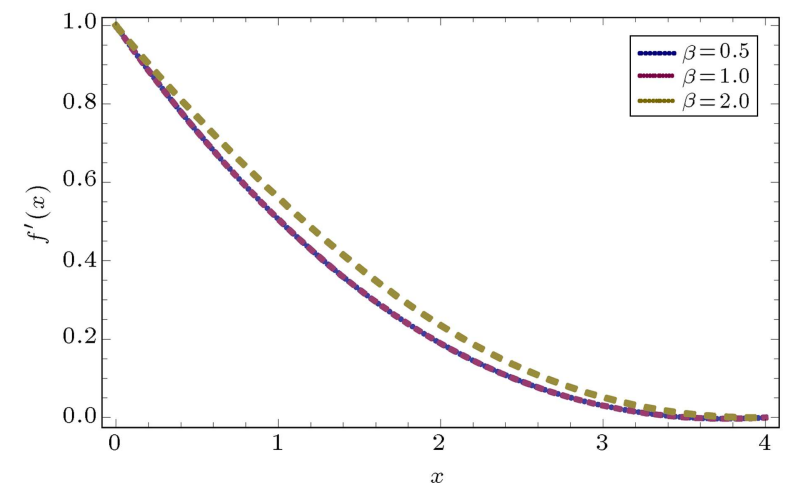

Figure 4. Effects of the Deborah number on $f^{\prime}(x)$ when $M=0.5$.

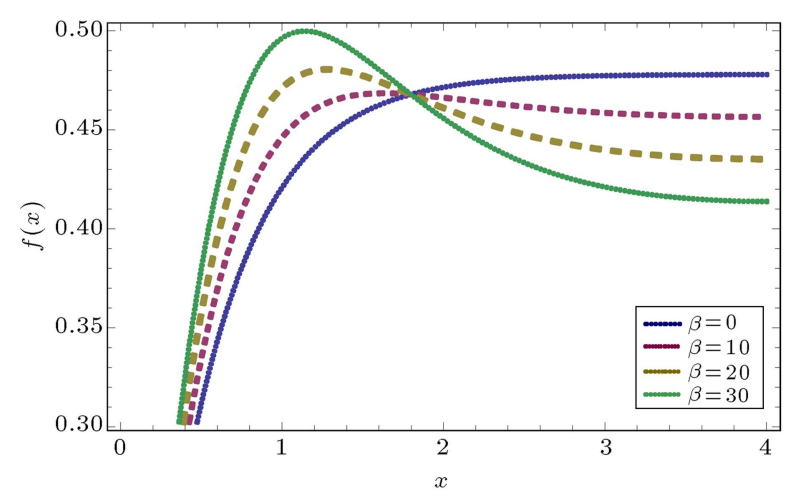

Figure 5. Effects of the Deborah number on $f(x)$ when $M=2$.

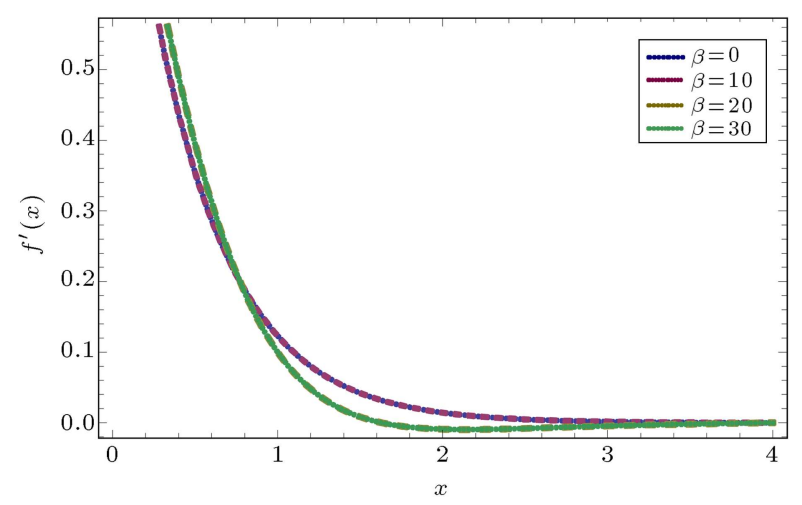

Figure 6. Effects of the Deborah number on $f^{\prime}(x)$ when $M=2$.

different values of $\beta$ at constant value of $M=0.5$. For small values of the Deborah number, the velocity profile has greater values and for large Deborah numbers, the velocity profile has smaller values. Figures 5 and 6 show that at high Hartman numbers with enlarged values of Deborah number, the velocity profile $f(x)$ at low Deborah numbers increases and at high Deborah numbers firstly increases and then decreases. Also, the velocity profile $f^{\prime}(x)$ at low Deborah numbers decreases and at high Deborah numbers firstly decreases and then increases. The residual and order of convergence to the OHAM solution are plotted in Figures 7 and 8.

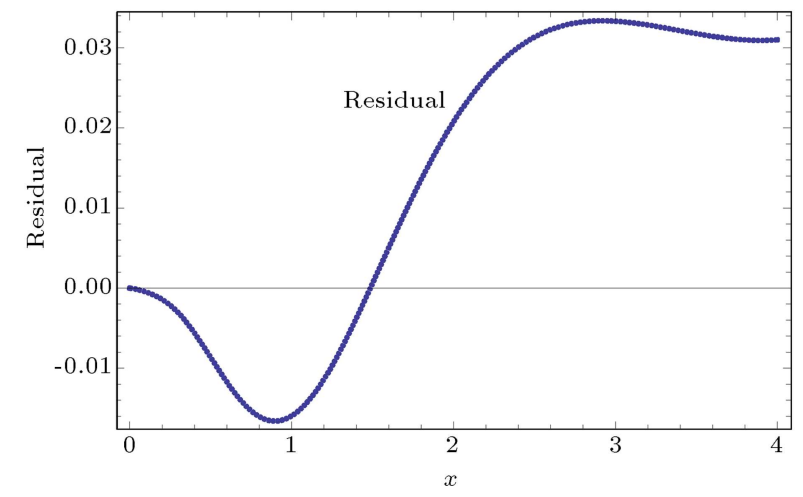

Figure 7. Residual plot for $\beta=0.5$ and $M=1$.

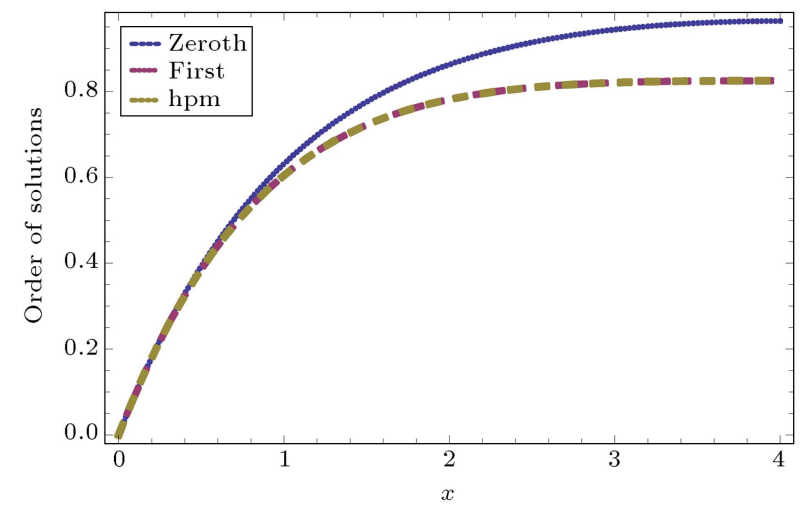

Figure 8. Order of solutions when $\beta=0.4$ and $M=1$.

\section{Conclusion}

In this work, we have seen effectiveness of OHAM [1115] for MHD boundary layer flow of an incompressible upper-convected Maxwell fluid. By applying the basic idea of OHAM to MHD boundary layer flow of an Incompressible Upper-Convected Maxwell Fluid, we found it simpler in applicability and more convenient to control convergence; also, it contained less computational overhead. Therefore, OHAM shows its validity and great potential for the solution MHD boundary layer flow of incompressible upper-convected Maxwell fluid problems arising in science and engineering. It has been observed that with the increase of Deborah number, the velocity profiles $f(x)$ and $f^{\prime}(x)$ steadily decreased and when increasing the Hartman number, thickness of the boundary layer decreased. The effect of Deborah number on the velocity profiles has been observed for low Hartman numbers $0 \leq M \leq 0.5$ and for high Hartman numbers $M>0.5$. It has been observed that the velocity profiles at low Hartman numbers are different from those at high Hartman numbers. The accuracy of OHAM results increases by increasing the order of approximation.

\section{Nomenclature}




$\begin{array}{ll}\mu & \text { Viscosity } \\ A_{1} & \text { Rivlin-Ericksen tensor } \\ p & \text { Pressure } \\ F_{\mathrm{L}} & \text { Lorentz force } \\ B & \text { Magnetic flux } \\ E & \text { Electric field } \\ S & \text { Extra stress tensor } \\ \lambda & \text { Relaxation time } \\ \rho & \text { Density } \\ f_{b} & \text { Body force } \\ J & \text { Electric current density } \\ \sigma & \text { Electric conductivity }\end{array}$

\section{References}

1. Chowdhury, S.H. "A comparison between the modified homotopy perturbation method and Adomian decomposition method for solving nonlinear heat transfer equations", J. Appl. Sci., 11, pp. 1416-1420 (2011).

2. Ganji, D.D., Afrouzi, G.A. and Talarposhti, R.A. "Application of variational iteration method and homotopy perturbation method for nonlinear heat diffusion and heat transfer equations", Phys. Lett. A, 368, pp. 450457 (2007).

3. Parsa, A.B., Rashidi, M.M., Bég, O.A. and Sadri, S.M. "Semi-computational simulation of magnetohemodynamic flow in a semi-porous channel using optimal homotopy and differential transform methods", Comp. Biol. Medi., 43, pp. 1142-1153 (2013).

4. He, J.H. "Homotopy perturbation technique", Comp. Math. Appl. Mech. Eng., 178, pp. $257-262$ (1999).

5. Franke, C. and Schaback, R. "Solving partial differential equations by collocation using radial basis functions", Appl. Math. Comp., 93, pp. 73-82 (1998).

6. Bellman, R. "Perturbation techniques in mathematics", Phys. Eng. Holt. Rin. Win., New York (1964).

7. Cole, J.D., Perturbation Methods in Applied Mathematics, Blai. Walt. MA. (1968).

8. O'Malley, R.E., Introduction to Singular Perturbation, Acad. Pres., New York (1974).

9. Liu, G.L. "New research direction in singular perturbation theory: artificial parameter approach and inverse perturbation technique", Conf. 7th Mod. Math. Mech. (1997).

10. Liao, S.J. "The proposed homotopy analysis technique for the solution of nonlinear problems", PhD Thesis, Shanghai University (1992).

11. Marinca, V., Herisanu, N. and Nemes, I. "Optimal homotopy asymptotic method with application to thin film flow", Cen. Euro. J. Phys., 6, pp. 648-53 (2008).

12. Marinca, V. and Herisanu, N. "Application of optimal homotopy asymptotic method for solving nonlinear equations arising in heat transfer", Int. Com. Heat Mas. Tran., 35, pp. 710-715 (2008).
13. Marinca, V. and Herisanu, N. "An optimal homotopy asymptotic method applied to the steady flow of a fourth-grade fluid past a porous plate", Appl. Math. Letts., 22, pp. 245-51 (2009).

14. Marinca, V., Herisanu, N. and Nemes, I. "A new analytic approach to nonlinear vibration of an electrical machine", Proc. Roma. Acad., 9, pp. 229-236 (2008).

15. Marinca, V. and Herisanu, N. "Determination of periodic solutions for the motion of a particle on a rotating parabola by means of the optimal homotopy asymptotic method", J. Sound. Vibr., 11, pp. 001-005 (2009).

16. Khater, A.H., Callebaut, D.K. and Abdelhameed, T.N. "Potential symmetry and invariant solutions of Fokker Planck equation in cylindrical coordinates related to magnetic field diffusion in magneto hydrodynamics including the Hall current", J. Eur. Phys., 50, pp. 1721 (2006).

17. Fetecau, C. "The Rayleigh Stokes problem for a fluid of Maxwellian type", Int. J. Non-Linr. Mech., 38, pp. 603-607 (2003).

18. Fetecau, C. "Starting solutions for some unsteady unidirectional flows of a second grade fluid", Int. J. Eng. Sci., 43, pp. 781-789 (2005).

19. Sadeghy, K., Najafi, A.H. and Saffaripour, M. "Sakiadis flow of an upper-convected Maxwell fluid", Int. J. Non-Linr. Mech., 40, pp. 1220-1228 (2005).

20. Ullah, H., Islam, S., Idrees, M. and Arif, M. "Solution of boundary layer problems with heat transfer by optimal homotopy asymptotic method", Abst. App. Anal. Arti., ID 324869, 10 pages (2013).

21. Ullah, H., Islam, S., Idrees, M. and Nawaz, R. "Optimal Homotopy Asymptotic Method to doubly wave solutions of the coupled Drinfel'd-Sokolv-Wilson equations", Math. Prob. Engg. Arti., ID 362816, 8 pages (2013).

22. Ullah, H., Islam, S., Idrees, M. and Fiza, M. "Solution of the differential-difference equations by optimal homotopy asymptotic method". Abst. Appl. Anal. Volu. Arti., ID 520467, 7 pages (2014).

23. Ullah, H., Islam, S., Sharidan, S. and Khan, I. "Formulation and application of optimal homotopy asymptotic method for coupled differential difference equations", Plos One, 10.1371/j.pone.0120127 (2015).

24. Rashidi, M.M., Laraqi, N. and Parsa, A.I. "Analytical modeling of heat convection in magnetized micropolar fluid by using modified differential transform method", Heat. Tran.-Asian Rese., 40, pp. 187-204 (2011).

25. Rahimi, M., Majidyfar, S. and Ganji, D.D. "Analytical and numerical analysis of MHD boundary layer flow of an incompressible upper convected Maxwell fluid", Int. J. Eng. Sci. Tech., 2, pp. 6909-6917 (2010).

26. Kolawole, S., Adegbie, J., Omowaye, D., Disu, A.B. and Animasaun, I.L. "Heat and mass transfer of upper convected Maxwell fluid flow with variable thermo- 
physical properties over a horizontal melting surface", App. Math., 6, pp. 1362-1379 (2015).

27. Ebrahimian, M., Khaki, M. and Abbasi, M. "Analytical solution of a transverse magnetic field on UCM fluids flow and species transfer with porous medium passing through channel", IJISET, 2(4) (April, 2015).

28. Hayat, T. and Sajid, M. "Homotopy analysis of MHD boundary layer flow of an upper convected Maxwell fluid", Int. J. Eng. Scie., 45, pp. 393-401 (2007).

29. Plonsey, R. and Collin, R.E., Principles and Applications of Electromagnetic Fields, 2th Edn., McGrawHill, New York (1982).

30. Wang, Y., Hayat, T. and Hutter, K. "On nonlinear magneto hydrodynamic problems of an Oldroyd 6 constant fluid", Int. J. Non-linear Mech., 40, pp. 49-58 (2005).

\section{Biographies}

Hakeem Ullah completed his MS in Mathematics at International Islamic University and $\mathrm{PhD}$ at Abdul Wali Khan University (AWKUM) in Pakistan. Dr. Ullah has published more than 30 articles in well reputed journals and is working as Assistant Professor at AWKUM.
Saeed Islam completed his MS in Mathematics at Quaid-e-azam University in Pakistan and $\mathrm{PhD}$ in China. Dr. Islam is working as Associate Professor and Chairman of the Department of Mathematics at Abdul Wali Khan University Mardan. He has published more than 200 articles in well reputed journals.

Ilyas Khan completed his MS in Mathematics at Quaid-e-azam University in Pakistan, PhD and Postdoctoral at University Technology Malaysia. Dr. Khan is working as Assistant Professor at Majmmah University in Saudia Arabia. He has published more than 100 articles in well reputed journals.

Sharidan Shafie Khan completed MS, PhD, and PostDoc in Mathematics at University Technology Malaysia. Dr. Shafie is working as Associate Professor at University Technology Malaysia. He has published more than 200 articles in well reputed journals.

Mehreen Fiza completed her MS in Mathematics and is doing his PhD in Mathematics at Abdul Wali Khan University in Pakistan. Ms. Fiza is working as lecturer in the Department of Mathematics at AWKUM and has more than 20 articles in well reputed journals. 